\title{
SOME DEFINABLE GALOIS THEORY AND EXAMPLES
}

\author{
OMAR LEÓN SÁNCHEZ AND ANAND PILLAY
}

\begin{abstract}
We make explicit certain results around the Galois correspondence in the context of definable automorphism groups, and point out the relation to some recent papers dealing with the Galois theory of algebraic differential equations when the constants are not "closed" in suitable senses. We also improve the definitions and results on generalized strongly normal extensions from [Pillay, "Differential Galois theory I", Illinois Journal of Mathematics, 42(4), 1998], using this to give a restatement of a conjecture on almost semiabelian $\delta$-groups from [Bertrand and Pillay, "Galois theory, functional Lindemann-Weierstrass, and Manin maps", Pacific Journal of Mathematics, 281(1), 2016].
\end{abstract}

§1. Introduction. We begin this introduction with some comments for the general logician, explaining what the paper is about and putting it in context.

The key notions are Galois theory and the Galois correspondence. The basic example is taking a field $K$ (such as the rationals) and adjoining to it all roots of some polynomial over $K$ in one variable, to generate a field $L$ say. $L$ is then said to be a Galois extension of $K$. The (finite) group $G$ of automorphisms of $L$ which fix $K$ pointwise is called the Galois group of $L$ over $K$ and the Galois correspondence is between subgroups of $G$ and fields in between $K$ and $L$. The paper [16] puts this into a more general model theoretic framework, following Poizat [23]. Another example, closer to the topic of this paper is when $K$ is a differential field (i.e., a field equipped with a derivation $\partial$, such as the field of rational functions $f(t)$ over a field $k$ together with the derivation $d / d t)$ and in place of a polynomial equation over $K$ we are given a linear differential equation over $K: \partial^{(n)} y+a_{n-1} \partial^{(n-1)} y+\cdots+a_{1} \partial y+a_{0} y=0$. Rather than adjoining "all solutions" to $K$ we want to adjoin a so-called fundamental system of solutions (i.e., a basis for the $n$-dimensional vector space of "all solutions"), to form a differential field $L$ such that the field of constants of $L$ is the same as the field of constants of $K$. Recall that the field of constants of $K$ is $C_{K}=\{a \in K: \partial a=0\}$, the constant field of $L$ is defined similarly. Here by "all solutions" we mean the set of solutions in a fixed differentially closed field extending $K$. The differential field $L$ is called a Picard-Vessiot extension of $K$. When the field of constants $C_{K}$ of $K$ is algebraically closed, this can be accomplished.

Received July 14, 2016.

2010 Mathematics Subject Classification. 03C60, 12H05.

Key words and phrases. model theory, differential fields, strongly normal extensions. 
Moreover, in this case the group $G$ of automorphisms of $L$ over $K$ (as differential fields), which may no longer be finite, is naturally isomorphic to a subgroup of $G L_{n}\left(C_{K}\right)$ defined by polynomial equations (a so-called linear algebraic group), where $G L_{n}\left(C_{K}\right)$ denotes the group of $n \times n$ matrices of nonzero determinant, with entries from $C_{K}$. The Galois correspondence is between algebraic subgroups of $G$ and differential fields in between $K$ and $L$. The same paper of Poizat referred to above gives a model theoretic account of this Picard-Vessiot Galois theory, making use of the first order theory $\mathrm{DCF}_{0}$ of differentially closed fields of characteristic zero, among other things. There are many variants of the Picard-Vessiot theory, which are detailed below. For example, we can consider a field $K$ equipped with two commuting derivations, sometimes called $\partial_{x}$ and $\partial_{t}$, and a linear differential equation over $K$ involving only the derivation $\partial_{x}$. When the field $C_{K}$ of $\partial_{x}$-constants of $K$ is differentially closed as a $\partial_{t}$-field, one again obtains the analogous existence of a Picard-Vessiot extension $L$ of $K$, a Galois group $G$, and a Galois correspondence, except that now $G$ is a subgroup of $G L_{n}\left(C_{K}\right)$ defined by differential equations involving $\partial_{t}$.

Several papers and/or preprints have appeared recently, giving a version of the Galois correspondence when the "constants" of the base field are not necessarily "closed" in the appropriate senses. The point of our current paper is to explain how these results are implicit or explicit in the model-theoretic literature: see [8, Section B.4] by Hrushovski or [9, Section 2.2] by Kamensky. The type of general model-theoretic results that we are referring to appear as parts (iii), (iv), and (v) of Theorem 2.3 below, a certain "indirect" Galois correspondence in the context of definable automorphism groups in first order theories.

In fact in the special case of "strongly normal" extensions of a differential field $K$ whose field of constants $C_{K}$ is not necessarily algebraically closed, the results are already in Kolchin's book [10, Chapter VI, Theorem 3]. In all these cases some translation between different languages is required, and the model-theoretic references may be unknown or rather obscure, at least for differential algebraists.

At this point we assume a little more familiarity with model theory and the relevant pieces of algebra (see the beginning of Section 3 for some details on the model theoretic context we have in mind; i.e., differentially closed fields).

The reason we talk about indirect Galois correspondences is as follows: In usual Galois theory, we take for $K$ say a perfect field, $P(x)=0$ a polynomial equation over $K$, and $L$ a splitting field for this equation. We can consider the (finite) set $Y$ of solutions of the equation in the algebraic closure $K^{\text {alg }}$ of $K$. Then $L$ is generated over $K$ by $Y$, and moreover the (finite) group of permutations of $Y$ which preserve all polynomial relations over $K$ coincides with $\operatorname{Aut}(L / K)$. The Galois correspondence is directly between subgroups of $\operatorname{Aut}(L / K)$ and fields in between $K$ and $L$. This picture also holds for the Picard-Vessiot theory when the constants are algebraically closed. Namely we take a linear differential equation $\delta y=A y$ (in vector form) over a differential field $(K, \delta)$ of characteristic zero with algebraically closed field $C_{K}$ of constants. Here, in 'vector form' means that we are viewing $y$ as an 
$n \times 1$ vector of variables and $A$ as an $n \times n$ matrix with entries in $K$ for some $n$. We now let $Y$ be the solution set of the equation in the differential closure $K^{\text {diff }}$ of $K$, that is $Y=\left\{b \in\left(K^{\text {diff }}\right)^{n}: \delta b=A b\right\}$, which is an $n$-dimensional vector space over the field $C=C_{K^{\text {diff }}}$ of constants of $K^{\text {diff }}$. We choose a basis $\bar{b}=\left\{b_{1}, \ldots, b_{n}\right\}$ of $Y$ over $C$, a so-called fundamental system of solutions, and let $L$ be the field generated over $K$ by $\bar{b}$ (which is in fact a differential field as $\delta b_{i}=A b_{i}$ for $i=1, \ldots, n$ ), the so-called Picard-Vessiot extension of $K$ for the equation $\delta y=A y$. It follows from $C_{K}$ being algebraically closed that actually $C=C_{K}$. Hence the full solution set $Y$ is contained in $L$, and the group of permutations of $Y$ preserving all differential polynomial relations over $K$, which has the structure of an algebraic subgroup $G$ of $G L_{n}(C)$, coincides with $A u t_{\delta}(L / K)$ the group of automorphisms as differential fields. The Galois correspondence is between algebraic subgroups of $G=A u t_{\delta}(L / K)$ and differential fields in between $K$ and $L$, as in the polynomial case.

But let us consider now the differential situation when the field of constants $C_{K}$ of $K$ is not necessarily algebraically closed. Then $C$, which is in fact equal to the algebraic closure of $C_{K}$ (see [15, Chapter 2, Lemma 2.11]), may properly contain $C_{K}$. The set $Y$ of solutions of the equation in $K^{\text {diff }}$ is still an $n$-dimensional vector space over $C$, and the group $G=A u t_{\delta}(Y / K, C)$ of permutations of $Y$ which preserve differential polynomial relations over $K$ and $C$ is an algebraic subgroup of $G L_{n}(C)$ as before. It may happen that there is a basis $\bar{b}$ of $Y$ over $C$ such that the differential field $L=K(\bar{b})$ has the same constants as $K$, in which case $L$ is called a Picard-Vessiot extension of $K$ for the equation. The existence of such $L$ implies that $G$ is defined over $C_{K}$, and we have moreover that $G$ coincides with $A_{u t}(L(C) / K(C))$ while $G\left(C_{K}\right)$, the $C_{K}$-rational points of $G$, coincides with $A u t_{\delta}(L / K)$. There is in general no direct Galois correspondence between algebraic subgroups of $G\left(C_{K}\right)$ and differential fields in between $K$ and $L$. But there is rather a Galois correspondence between algebraic subgroups of $G$, defined over $C_{K}$, and differential fields in between $K$ and $L$ : given such an intermediate differential field $F$, the elements of $G$ which fix $F$ pointwise form an algebraic subgroup of $G$ defined over $C_{K}$, etc. This is an example of the indirect Galois correspondence, which is given by the existence of a Picard-Vessiot extension, and which is a special case of the model theoretic result we will be presenting. Let us mention a simple example of a Picard-Vessiot extension where the Galois correspondence is not direct:

Example 1.1. Let $K=\mathbb{Q}$ and $L=\mathbb{Q}\left(e^{x}\right)$, with derivation $\delta=d / d x$. Then $L$ is a Picard-Vessiot extension of $K$ for the equation $\delta y=y$. It can be seen that in this case $G=\mathbb{G}_{m}\left(C_{K^{d i f f}}\right)=\mathbb{G}_{m}\left(\mathbb{Q}^{\text {alg }}\right)$, the multiplicative group of $\mathbb{Q}^{\text {alg }}$. The intermediate differential fields are of the form $\mathbb{Q}\left(e^{n x}\right)$ as $n$ varies in $\mathbb{N}$. However, the only algebraic subgroups of $G\left(C_{K}\right)=\mathbb{G}_{m}(\mathbb{Q})$ are the two obvious ones and $\{-1,1\}$. Thus, the Galois correspondence cannot be direct. The indirect correspondence is given by mapping $\mathbb{Q}\left(e^{n x}\right)$ to the algebraic subgroup of $\mathbb{G}_{m}\left(\mathbb{Q}^{\text {alg }}\right)$ consisting of the $n$-th roots of unity when $n>0$, and, of course, $\mathbb{Q}$ maps to $\mathbb{G}_{m}\left(\mathbb{Q}^{\text {alg }}\right)$. 
The general model-theoretic context is roughly (working inside an ambient saturated structure $\bar{M}$ ): we are given a set $A$ of parameters, and $A$-definable sets $Y$ and $X$. Under suitable conditions $\operatorname{Aut}(Y / A, X)$, the group of permutations of $Y$ which are elementary over $A, X$ will have the structure of an $A$-definable group $G$. And under additional conditions we will study the "extension" $B / A$ where $B=\operatorname{dcl}(A, b)$ for a suitable $b \in Y$, as well as $\operatorname{Aut}(B / A)$, which are the general versions of a Picard-Vessiot extension $L / K$, and its automorphism group $A u t(L / K)$. And we will describe the relations between these objects as well as the (indirect) Galois correspondence. This will be accomplished in the next section, where the basic set-up (or definition) is given by conditions (I) and (II) and the main result is Theorem 2.3. As remarked in [8] what is being discussed is when and how the theory of definable automorphism groups translates to a theory of extensions $B / A$ of definably closed sets and their automorphism groups.

In the final section we show how this set-up and Theorem 2.3 subsume the various differential algebraic examples. One possibly novel thing on the differential algebraic side will be a refinement of the generalized strongly normal theory from [18,19], where we are able to drop the assumption that $X(K)=X\left(K^{\text {diff }}\right)$. We will use this to give a rather clearer statement of Conjecture 2.3 from [1], which is related to Ax-Lindemann-Weierstrass questions for (families of) semiabelian varieties. This is possibly the only part of the paper which requires some more background from the reader.

We should make it clear that our aim is not to give yet another exposition of definable automorphism groups and/or the model theoretic approach to the Galois theory of differential equations, of which there are many ([23], [24, Section 8.3], as well as the more specialized [21] and [20, Chapter 8, Section 4]), but rather to explain concisely how a number of recent results in the literature follow from general and known model-theoretic considerations.

$\S 2$. Model theoretic context and results. Throughout this section we assume familiarity with the basics of model theory (the material in [15, Chapter 1] covers most of what we use here). Let us fix a complete theory $T$ in the language $L . \bar{M}$ denotes a saturated model of $T$, and A, B, ... small subsets of $\bar{M}$. For convenience we assume

(†) $\quad T$ is $\omega$-stable and $T=T^{e q}$.

In place of the $T=T^{e q}$ assumption, the reader could just assume that $T$ is 1-sorted with elimination of imaginaries, which will actually be the case in the differential algebraic contexts and examples, where $T=\mathrm{DCF}_{0, m}$.

We fix a definably closed set $A$, a complete type $q(x)$ over $A$, and an $A$-definable set $X$. We consider the following conditions on $q$ and $X$.

(I) For any realizations $b, b^{\prime}$ of $q, b^{\prime} \in \operatorname{dcl}(b, X, A)$.

(II) For any tuple $c$ from $X, q(x)$ and $r(y)=t p(c / A)$ are weakly orthogonal, namely $q(x) \cup r(y)$ extends to a unique complete $x y$-type over $A$.

We do not really wish to add new and unnecessary terminology to the subject, but we mention some possibilities in the following remark. 
REMARK 2.1.

(1) Condition (I) says that $q$ is internal to $X$ and is already the type of a "fundamental system of solutions". We could use the expression " $q$ is strongly internal to $X$ " for (I).

(2) Condition (II) says that for any two realizations $b_{1}, b_{2}$ of $q$ in $\bar{M}$,

$$
t p\left(b_{1} / A, X\right)=t p\left(b_{2} / A, X\right),
$$

which has to be the unique nonforking extension of $q$ over $A, X$.

(3) We might also want to express conditions (I) and (II) by " $B$ is a Galois extension of $A$, relative to $X^{\prime \prime}$, where $B=\operatorname{dcl}(A, b)$ and $b$ realizes $q$.

LEMMA 2.2.

(i) Let $b$ realize $q$ and let $B=\operatorname{dcl}(A, b)$. Then $q$ and $X$ satisfy condition (II) above if and only if

(II') $\operatorname{dcl}(A, X) \cap B=A$.

(ii) Suppose (I) and (II) hold (for $q, X)$. Then $q$ is isolated.

Proof. (i) (II) implies (II') is clear. For the converse: By $\omega$-stability there is $c \in \operatorname{dcl}(A, X)$ such that $\operatorname{tp}(b / A, X)$ is definable over $c$ and note that $c \in \operatorname{dcl}(b, A)$. So $c \in \operatorname{dcl}(A, X) \cap B$. By (II'), $c \in A$, which means that $\operatorname{tp}(b / A, X)$ is definable over $A$. Note that for any other realization $b^{\prime}$ of $q$ there is an $A$-automorphism of $\bar{M}$ taking $b$ to $b^{\prime}$ and fixing $X$ setwise, hence $\operatorname{tp}\left(b^{\prime} / A, X\right)$ is definable over $A$ by the same schema defining $t p(b / A, X)$, and hence $t p\left(b^{\prime} / A, X\right)=t p(b / A, X)$, giving (II).

(ii) By (I) and compactness there is a formula $\phi(x) \in q(x)$ and a partial $A$-definable function $f(-,-)$ such that for all $b, b^{\prime}$ satisfying $\phi(x)$, there is a tuple $c$ of elements of $X$ such that $f(b, c)=b^{\prime}$. Now, by $\omega$-stability, let $M_{0}$ be a prime model over $A, X$, and let $b$ realize $\phi(x)$ in $M_{0}$ and let $b^{\prime}$ be any realization of $q$ in $\bar{M}$. By what we just said there is $c$ from $X$ such that $f(b, c)=b^{\prime}$. As $t p(b / A, X)$ is isolated, so is $t p\left(b^{\prime} / A, X\right)$. But by (II), $q$ has a unique extension to a complete type over $A, X$, which must be $t p\left(b^{\prime} / A, X\right)$. By compactness $q$ is isolated.

We are still fixing $A, q$, and $X$. Let $Q$ be the set of realizations of $q$ (in $\bar{M})$. Let $\operatorname{Aut}(Q / X, A)$ denote the group of permutations of $Q$ which are elementary over $A, X$, namely preserve all relations (on arbitrary $Q^{n}$ ) which are $A, X$-definable. Equivalently (using stability), $A u t(Q / X, A)$ is the group of permutations of $Q$ induced by automorphisms of $\bar{M}$ which fix pointwise $A, X$. Let us fix a realization $b$ of $q$, and let $B=\operatorname{dcl}(A, b)$. Let $\operatorname{Aut}(B / A)$ denote the group of permutations of $B$ which are elementary over $A$ in the sense of $\bar{M}$. With this notation we have:

THEOREM 2.3. Suppose that $q$ and $X$ satisfy conditions (I) and (II). Then

(i) $\operatorname{Aut}(Q / A, X)$ acts regularly (in particular transitively) on $Q$. Indeed every $\sigma \in \operatorname{Aut}(Q / A, X)$ is determined by $\sigma\left(b^{\prime}\right)$ for some/any $b^{\prime} \in Q$ and all elements of $Q$ have the same type over $A, X$.

(ii) There is an A-definable group $G$ whose domain is an A-definable set contained in $\operatorname{dcl}(A, X)$, and a group isomorphism $\mu$ between $A u t(Q / A, X)$ and $G$ such that the induced action of $G$ on $Q$ is $B$-definable. 
(iii) $\mu$ induces an isomorphism between $A u t(B / A)$ and $G(A)$, the group of elements of $G$ which are in $A$.

(iv) There is a Galois correspondence between definably closed sets in between $A$ and $B$ and $A$-definable subgroups of $G$. More precisely, for a definably closed set $C$ with $A \subseteq C \subseteq B$, if we let

$$
H_{C}=\{\sigma \in G: \sigma \text { fixes } C \text { pointwise }\} \text {, }
$$

then $H_{C}$ is an $A$-definable subgroup of $G$, every $A$-definable subgroup of $G$ appears as some $H_{C}$, and $C \subseteq C^{\prime}$ iff $H_{C^{\prime}} \leq H_{C}$. In addition:

(v) Let $C$ be a definably closed set with $A \subseteq C \subseteq B$. Then, $t p(b / C)$ and $X$ also satisfy conditions (I) and (II), and $H_{C}$ is isomorphic to Aut $\left(Q_{C} / C, X\right)$, where $Q_{C}$ is the set of realizations of $t p(b / C)$. Moreover, there is a finite tuple e such that $C=\operatorname{dcl}(e, A)$, and the type $t p(e / A)$ satisfies $(\mathrm{I})$ if and only if $H_{C}$ is a normal subgroup of $G$. In this case, $G / H_{C}$ is isomorphic to $\operatorname{Aut}(P / A, X)$ where $P$ is the set of realizations of $t p(e / A)$.

ProOF. Everything is explicit or implicit in the literature (see for instance $[7,8,25]$ ), but we give a quick proof of the theorem in its entirety. (i) and (ii) set up the objects, and the main points are (iii), (iv), and (v).

The proof of (i) is contained in the statement.

(ii) In Lemma 2.2 we saw that

(*) $Q$ is an $A$-definable set,

and moreover by (II) (and the proof of Lemma 2.1(i))

(**) $\quad q$ has a unique extension to a complete type over $A, X$ which is moreover definable over $A$.

By (I) and compactness, there is an $A$-definable (partial) function $f_{0}$ such that every element of $Q$ is of the form $f_{0}(b, c)$ for some tuple $c$ (of fixed length) from $X$. Let $Y_{0}$ be the set of tuples of this fixed length from $X$ such that $f_{0}(b, c) \in Q$. By $\left(^{*}\right)$ and $(* *) Y_{0}$ is an $A$-definable set of tuples from $X$. Let $E$ be the equivalence relation on $Y_{0}$ defined by $E\left(c_{1}, c_{2}\right)$ iff $f_{0}\left(b, c_{1}\right)=f_{0}\left(b, c_{2}\right)$. Then, again by $(* *), E$ is $A$-definable. Hence the set $Y_{0} / E$ which is $A$-definable in $\bar{M}^{e q}$ is contained in $\operatorname{dcl}(A, X)$. Let $Y$ denote $Y_{0} / E$. We can clearly rewrite $f_{0}$ as an $A$-definable map from $Q \times Y$ to $Q$ and now note that for any $b^{\prime} \in Q$ there is a unique $d \in Y$ such that $b^{\prime}=f(b, d)$.

For $\sigma \in \operatorname{Aut}(Q / A, X)$ let $\mu(\sigma)$ be the unique element of $Y$ such that $\sigma(b)=f(b, \mu(\sigma))$. The map $\mu$ sets up a bijection between $A u t(Q / A, X)$ and $Y$. Using (**) one sees that the group operation on $Y$ induced by $\mu$ is $A$-definable, and the induced action on $Q$ is $B$-definable. We let $G$ denote the set $Y$ equipped with this group structure. So $G$ is an $A$-definable group and its action on $Q$ is definable over $B$. We will often identify $G$ with $A u t(Q / A, X)$.

(iii) As mentioned above $G$ lives on the $A$-definable set $Y$, so $G(A)$ is, as a set, the collection of elements of $Y$ which are in $\operatorname{dcl}(A)=A$. The nature of our identification of $G$ with $\operatorname{Aut}(Q / X, A)$ yields that $G(A)$ consists of those $\sigma \in \operatorname{Aut}(Q / X, A)$ such that $\sigma(b) \in B$. Here is the explanation: $\sigma \in G(A)$ means $\mu(\sigma) \in A$ which implies $\sigma(b)=f(b, \mu(\sigma)) \in B$. Conversely, if 
$\sigma(b) \in B$ then as $\mu(\sigma)$ is determined by $b$ and $\sigma(b)$, also $\mu(\sigma) \in B$. But $\mu(\sigma) \in Y \subset d c l(A, X)$, hence by Lemma 2.2 and condition (II), $\mu(\sigma) \in A$, as required. Note finally that we can view $\operatorname{Aut}(B / A)$ as precisely the subgroup of $\operatorname{Aut}(Q / X, A)$ consisting of those $\sigma$ such that $\sigma(b) \in B$. This completes the proof.

One should note that as by (II)', $d \operatorname{cl}(A, X) \cap B=d c l(A, X) \cap A, G(B)$ coincides with $G(A)$.

(iv) For the Galois correspondence the main point is that condition (II) yields that

$$
\text { (***) for } D_{1}, D_{2} \subseteq B, \quad D_{2} \subseteq \operatorname{dcl}\left(D_{1}, A\right) \text { iff } D_{2} \subseteq \operatorname{dcl}\left(D_{1}, A, X\right) .
$$

Note that the latter is equivalent to " $D_{2}$ is fixed by every $\sigma \in G$ which fixes $D_{1}$ ". Moreover, for $d \in B$

$$
(* * * *) \quad\{\sigma \in G: \sigma(d)=d\} \text { is an } A \text {-definable subgroup of } G .
$$

This is because if $d=h(b)$ for $h$ an $A$-definable function, then $\sigma \in G$ fixes $d$ iff $h(\sigma(b))=h(b)$ iff $h(f(b, \sigma))=h(b)$, which is, by $\left({ }^{* *}\right)$, an $A$-definable condition on $\sigma$. Now let $C$ be an arbitrary subset of $B$ and $H_{C}$ the set of $\sigma \in G$ which fix $C$ pointwise. By (****) and $\omega$-stability, $H_{C}$ is an $A$ definable subgroup of $G$ and, by (***), the set of elements of $B$ which are fixed by $H_{C}$ is precisely $\operatorname{dcl}(A, C)$.

On the other hand, suppose $H$ is an arbitrary subgroup of $G$ and let $C$ be the set of elements of $B$ fixed pointwise by every element of $H$. Then, as the elements of $H$ also fix $A$-pointwise, it follows that $C$ is a definably closed subset of $B$ containing $A$. Moreover, by the above, $H_{C}$ is an $A$-definable subgroup of $G$ containing $H$.

(v) Let $q_{C}=t p(b / C)$, and let $Q_{C}$ denote its set of realizations. As $q_{C}$ extends $q$, (I) holds for $q_{C}$ and $X$. As $B=d c l(A, b), t p(B / A)$ has a unique extension over $A, X$, so $t p(b C / A)$ has a unique extension over $A, X$, whereby $t p(b / C)$ has a unique extension over $C \cup X$. So $q_{C}$ and $X$ satisfy (II).

As remarked earlier any $\sigma \in \operatorname{Aut}(Q / A, X)$ is determined by the value $\sigma(b)$. Hence $\{\sigma \in \operatorname{Aut}(Q / A, X): \operatorname{tp}(\sigma(b) / C)=t p(b / C)\}$ is a subgroup of $\operatorname{Aut}(Q / A, X)$ which is naturally isomorphic to $\operatorname{Aut}\left(Q_{C} / A, X\right)$. Moreover the image of this subgroup under $\mu$ is clearly $H_{C}$.

As $C$ is a definably closed subset of $\operatorname{dcl}(A, b), \omega$-stability implies that $C=\operatorname{dcl}(A, e)$ for some finite tuple $e \in C$. Note that $p=t p(e / A)$ (and $X$ ) automatically satisfy condition (II). Suppose that $t p(e / A)$ and $X$ satisfy (I). Let $\sigma \in \operatorname{Aut}\left(Q_{C} / A, X\right)$ and $\tau \in \operatorname{Aut}(Q / A, X)$. Let $e^{\prime}=\tau(e)$. So by (I), $e^{\prime} \in \operatorname{dcl}(A, e, X)$, whereby $\sigma\left(e^{\prime}\right)=e^{\prime}$. Hence $\sigma^{-1} \tau \sigma(e)=e$, so $\sigma^{-1} \tau \sigma$ is in $\operatorname{Aut}\left(Q_{C} / A, X\right)$. We have shown that $\operatorname{Aut}\left(Q_{C} / A, X\right)$ is normal in $\operatorname{Aut}(Q / A, X)$, whence $H_{C}$ is normal in $G$. Conversely, if $A u t\left(Q_{C} / A, X\right)$ is normal in $\operatorname{Aut}(Q / A, X)$, then for any $\rho \in H_{C}$ and $\sigma \in G$ we have $\rho(\sigma(e))=\sigma(e)$. Therefore, all the elements of $H_{C}$ fix $P$ pointwise (the latter is the set of realizations of $p)$. Thus, $P \subseteq \operatorname{dcl}(e, A, X)$. This shows that $p$ and $X$ satisfy (I). Finally, the restriction to $P$ yields a group homomorphism from $G$ to $\operatorname{Aut}(P / A, X)$ with kernel $H_{C}$. 
We end this section with a couple of remarks. The first concerns the definability properties of $G(A)$. $G$ is of course a group definable in the ambient structure $\bar{M}$, with parameters from $A$. What about $G(A)$ which corresponds to $\operatorname{Aut}(B / A)$ ? We maintain the earlier assumptions, notation and conditions (I), (II), for $q, X$.

REMARK 2.4.

(1) Let $M_{0}$ be the prime model over $A$ (which exists by $\omega$-stability). Suppose that $X(A)=X\left(M_{0}\right)$, namely all points of $X$ in the model $M_{0}$ are already in $A$. Then $G(A)$ coincides with the interpretation $G\left(M_{0}\right)$ of the formula over $A$ defining $G$ in $\bar{M}$, in the elementary substructure $M_{0}$. So bearing in mind part (iii) of Theorem 2.3, we see in this case that $\operatorname{Aut}(B / A)$ is a definable group in the model $M_{0}$, and we easily obtain from Theorem 2.3 the Galois correspondence between subgroups of $G\left(M_{0}\right)$ definable in $M_{0}$ and definably closed sets in between $A$ and $B$.

(2) If the theory $T$ is one-sorted (with elimination of imaginaries) and has quantifier elimination, then $X$ will be a quantifier-free $A$-definable set in $\bar{M}$, hence as the universe of $G$ is $Y$ which will be an $A$-definable subset of some power of $X, G(A)$ will be a group (quantifier-free) definable (without parameters) in the structure whose universe is $X(A)$ and whose relations are intersections with $X(A)^{n}$ of $A$-definable subsets of $X^{n}$. See Example 1.1 in the introduction for the failure of the direct Galois correspondence in this situation.

In the next remark we point out an extension of part (iii) of Theorem 2.3, whose proof is left to the reader.

REMARK 2.5. Under the same assumptions and notation of Theorem 2.3, let $D$ be any definably closed set containing $B$, and let $B_{D}$ be $\operatorname{dcl}(b, A, X(D))$. Then $\mu$ induces an isomorphism between $A u t\left(B_{D} / A, X(D)\right)$ and $G(D)$.

§3. Applications to and interpretation in differential Galois theory. We now give the interpretation of the above model-theoretic results in the context of differential fields. In these examples we will start with the most concrete, ending with the most general, for pedagogical reasons.

We work in the language of differential rings with $m$ derivations

$$
\mathcal{L}_{\text {rings }} \cup\left\{\delta_{1}, \ldots, \delta_{m}\right\} .
$$

All model-theoretic and differential-algebraic terminology refers to this language. Recall that $\mathrm{DCF}_{0, m}$ is the model companion of the theory of fields of characteristic zero equipped with $m$ commuting derivations, and is called the theory of differentially closed fields of characteristic zero (with $m$ commuting derivations). It is well known that this theory is $\omega$-stable and eliminates imaginaries, so fits into the assumption $(\dagger)$. Moreover, it has quantifier elimination [17]. When $m=1$ (the so-called ordinary case) the theory is sometimes just denoted $\mathrm{DCF}_{0}$. In this ordinary case we write $\delta$ for $\delta_{1}$. 
The saturated model $\bar{M}$ is denoted

$$
\left(\mathcal{U}, \Pi=\left\{\delta_{1}, \ldots, \delta_{m}\right\}\right) \models \mathrm{DCF}_{0, m} .
$$

$K, L$, etc. denote (small) differential subfields $K$ of $\mathcal{U}$. For any set $A$ of tuples from $\mathcal{U}$, the definable closure of $A$ in $\mathcal{U}$ coincides with the differential field generated by the coordinates of the members of $A$, and the (modeltheoretic) algebraic closure of $A$ coincides with the field-theoretic algebraic closure of the differential field just mentioned. $K\langle A\rangle$ denotes the differential field generated over $K$ by $A$, where $A$ is a subset of $\mathcal{U}$. For any subset $\mathcal{D} \subseteq \Pi$ and any differential subfield $F$ of $\mathcal{U}$ we let $F^{\mathcal{D}}$ denote the subfield of $\mathcal{D}$-constants of $F$; that is,

$$
F^{\mathcal{D}}=\{a \in F: \delta(a)=0 \text { for all } \delta \in \mathcal{D}\} .
$$

We let $\mathcal{C}$ denote $\mathcal{U}^{\Pi}$; that is, $\mathcal{C}$ is the universal field of $\Pi$-constants of $\mathcal{U}$. Also, we write $C_{F}$ for $F^{\Pi}$ (the $\Pi$-constants of $F$ ). This is consistent with notation in the introduction.

Lemma 3.1. Suppose $K \subseteq$ L are differential fields (i.e., differential subfields of $\mathcal{U})$. Let $\mathcal{D}$ be a nonemptyset subset of $\Pi$, and let $X=\mathcal{U}^{\mathcal{D}}$. Then

(1) $\operatorname{dcl}(K, X) \cap L=K\left\langle L^{\mathcal{D}}\right\rangle$.

(2) $\operatorname{dcl}(K, X) \cap L=K$ means precisely that $K^{\mathcal{D}}=L^{\mathcal{D}}$.

Proof. (i) A consequence of the linear disjointness property of the constants (see [10, Chapter II, Section 1]) is that for any intermediate differential field $K \leq E \leq K\left\langle\mathcal{U}^{\mathcal{D}}\right\rangle$, we have that

$$
K\left\langle E^{\mathcal{D}}\right\rangle=E .
$$

Applying this with $E=\operatorname{dcl}(K, X) \cap L$ we get

$$
\operatorname{dcl}(K, X) \cap L=K\left\langle\mathcal{U}^{\mathcal{D}}\right\rangle \cap L=K\left\langle K\left\langle\mathcal{U}^{\mathcal{D}}\right\rangle \cap L^{\mathcal{D}}\right\rangle=K\left\langle L^{\mathcal{D}}\right\rangle .
$$

Note that in the second equality is where we used (3.1).

(ii) follows directly from (i).

3.1. Picard-Vessiot extensions. We focus first on the ordinary case, $m=1$. A linear differential equation over $K$ (in vector form) is something of the form $\delta y=A y$ where $y$ is a $n \times 1$ column vector of indeterminates and $A$ is an $n \times n$ matrix with coefficients from $K$. A fundamental system of solutions of the equation is a set $y_{1}, \ldots, y_{n}$ of solutions of the equation such that the determinant of the $n \times n$ matrix $\left(y_{1}, \ldots, y_{n}\right)$ is nonzero. Equivalently $\left(y_{1}, \ldots, y_{n}\right)$ is a basis over $\mathcal{C}$ of the $\mathcal{C}$-vector space $V$ of solutions to the equation in $\mathcal{U}$. By a Picard-Vessiot extension of $K$ for the equation $\delta y=A y$ is meant a differential field $L$ generated over $K$ by some fundamental system $\left(y_{1}, \ldots, y_{n}\right)$ of solutions, and such that $C_{L}=C_{K}$. For the basic results on $P V$ extensions and a bit of history on the subject we refer the reader to the expository paper [14]. We see from Lemma 3.1 that if $L$ is a PicardVessiot extension of $K$ for the equation then $L$ is of the form $\operatorname{dcl}(K, b)$, where $q=t p(b / K)$ and $\mathcal{C}$ satisfy conditions (I) and (II) from the previous section. Hence Theorem 2.3 holds in this specific context. In this case the construction or identification of the Galois group is more direct: for each $\sigma \in \operatorname{Aut}(Q / A, \mathcal{C}), \sigma(b)=b \mu(\sigma)$ for a unique matrix $\mu(\sigma) \in G L_{n}(\mathcal{C})$, and $\mu$ 
gives an isomorphism between $\operatorname{Aut}(Q / A, \mathcal{C})$ and an algebraic subgroup $G$ of $G L(n, \mathcal{C})$ defined over $C_{L}=C_{K}$. The Galois correspondence is between algebraic subgroups of $G$ defined over $C_{K}$ and differential fields in between $K$ and $L$. Theorem 2.3 in this context is contained in [10, Chapter VI] in the slightly more general form of strongly normal extensions. In any case what we have described here subsumes [5, Theorem 4.4] and [4].

The previous paragraph extends to the partial case $m>1$, where we now consider a set $\delta_{1} y=A_{1} y, \ldots, \delta_{m} y=A_{m} y$ of linear DE's over $K$ and where the matrices $A_{i}$ satisfy suitable integrability (or Frobenius) conditions, see [22, Appendix D].

3.2. Strongly normal extensions. We work with any $m \geq 1$. According to Kolchin [10] a strongly normal extension $L$ of a differential field $K$ is a differential field extension $L$ of $K$ such that $L$ is finitely generated over $K$ and for every automorphism $\sigma$ of $\mathcal{U}$ over $K, \sigma(L) \subseteq L\langle\mathcal{C}\rangle$ and $\sigma$ fixes $C_{L}$ pointwise. As Kolchin notes the condition that every automorphism of $\mathcal{U}$ over $K$ fixes $C_{L}$ is equivalent to requiring that $C_{L}=C_{K}$.

Let $L$ be generated over $K$ by the finite tuple $b$, and let $q=t p(b / K)$. Thus the condition for $L$ to be a strongly normal extension of $K$ means precisely that $q$ and $\mathcal{C}$ satisfy conditions (I) and (II) from Section 2 (using Lemma 3.1).

Hence Theorem 2.3 applies. Using elimination of imaginaries, stability, and the fact that $\mathcal{C}$ is an algebraically closed field without additional structure, the Galois group $G$ as described in the proof of Theorem 2.3, is the group of $\mathcal{C}$-points of an algebraic group defined over $C_{K}$. Again the Galois correspondence is between algebraic subgroups of $G$ defined over $C_{K}$ and differential fields in between $K$ and $L$. Theorem 2.3 is again contained in Kolchin, [10, Chapter VI]. In fact see Theorems 3 and 4 there for the Galois correspondence. This subsumes [2]. Indeed, part (iii) of Theorem 2.3 that $\operatorname{Aut}(L / K)$ is $G(A)$, includes, by virtue of Remark 2.4(2), the statement in [2, Theorem 3.15] that $A u t(L / K)$ is interpretable in the field $C_{K}$. In fact one actually obtains definability (rather than just interpretability). Similarly, Remark 2.5 includes the statement in [2, Corollary 3.21$]$ with definability in place of interpretability.

3.3. Parameterized Picard-Vessiot $(P P V)$ extensions. The context is a differential field $K$ equipped with $m+1$ commuting derivations which we write as $\delta_{x}, \delta_{t_{1}}, \ldots \delta_{t_{m}}$, and a linear differential equation $\delta_{x} y=A y$ over $K$. Here the derivations $\delta_{t_{1}}, \ldots, \delta_{t_{m}}$ are sometimes referred to as the parametric derivations (thinking of the $t_{i}$ 's as parameters), and hence the name parameterized Picard-Vessiot theory. Our universal domain $\mathcal{U}$ is a saturated model of $\mathrm{DCF}_{0, m+1}$, and the solution set $V$ of the equation is an $n$-dimensional vector space over $\mathcal{U}^{\delta_{x}}$, the field of $\delta_{x}$-constants of $\mathcal{U}$. A fundamental system of solutions is again a matrix $\left(y_{1}, \ldots, y_{n}\right)$ of solutions, with nonzero determinant, and by a $P P V$-extension of $K$ for the equation we mean a differential field $L$ generated over $K$ by such a fundamental system of solutions, such that also $K^{\delta_{x}}=L^{\delta_{x}}$. Note that when $m=0$, no parameters, we are in the situation of Section 3.1. If $\left(y_{1}, \ldots, y_{n}\right)$ is such and its type 
(in the language with all the derivations) over $K$ is $q$ then as before, $q$ and $\mathcal{U}^{\delta_{x}}$ satisfy conditions (I) and (II). The Galois group $G$ from Theorem 2.3 is a subgroup of $G L_{n}\left(\mathcal{U}^{\delta_{x}}\right)$ definable over $K^{\delta_{x}}$ in the differentially closed field $\left(\mathcal{U}^{\delta_{x}}, \delta_{t_{1}}, \ldots, \delta_{t_{m}}\right)$. The Galois correspondence (when $K^{\delta_{x}}$ is not necessarily differentially closed) was established in Section 8.1 of [6], but again is contained in Theorem 2.3. Let us give a basic example of a $P P V$ extension where the Galois correspondence is not direct (for details see [3, Example 3.1]):

ExAmple 3.2. Let $K=\mathbb{C}(x, t)$ be the differential field with derivations $\Pi=\left\{\delta_{x}=\frac{\partial}{\partial x}, \delta_{t}=\frac{\partial}{\partial t}\right\}$ where we view $t$ as the parameter. Consider the linear differential equation $\delta_{x} y=\frac{t}{x} y$ in the single variable $y$. If we let $L=K\left(x^{t}, \log x\right)$, then $L$ is a differential field extension of $K$, and it is in fact a $P P V$ extension. In this case the Galois group $G$ is the subgroup of $\mathbb{G}_{m}\left(\mathcal{U}^{\delta_{x}}\right)$ given by

$$
G=\left\{z \in \mathbb{G}_{m}\left(\mathcal{U}^{\delta_{x}}\right): \delta_{t}\left(\frac{\delta_{t} z}{z}\right)=0\right\} .
$$

Thus, the group $A u t_{\Pi}(L / K)$ of $\Pi$-automorphisms of $L$ fixing $K$ is given by $G\left(K^{\delta_{x}}\right)=G(\mathbb{C}(t))=\mathbb{G}(\mathbb{C})$. It then follows that $\log x$ is fixed by every element of $\operatorname{Aut}_{\Pi}(L / K)$, and hence there cannot be a direct Galois correspondence.

Let us mention that a strongly normal analogue of $P P V$ extensions was studied in [13] also establishing the (indirect) Galois correspondence.

3.4. Landesman's strongly normal extensions. Landesman [11] slightly generalizes Kolchin's definition of strongly normal extension by replacing $\mathcal{C}\left(=\mathcal{U}^{\Pi}\right)$ by $\mathcal{U}^{\mathcal{D}}$ for any nonempty subset $\mathcal{D}$ of $\Pi$. Formally he takes $\Delta$ to be $\Pi \backslash \mathcal{D}$ and defines $L$ to be a $\Delta$-strongly normal extension of $K$ if $L$ is finitely generated over $K$, and for any automorphism $\sigma$ of $\mathcal{U}$ over $K, \sigma(L) \subseteq L\left\langle\mathcal{U}^{\mathcal{D}}\right\rangle$ and $\sigma$ fixes $L^{\mathcal{D}}$ pointwise. As in our discussion of strongly normal extensions above, this is equivalent to requiring that $q, \mathcal{U}^{\mathcal{D}}$ satisfy conditions (I) and (II) from Section 2. In [11, Theorem 2.34] Landesman proves the Galois correspondence when $K^{\mathcal{D}}$ is differentially closed as a $\Delta$-field, and asks if the differentially closed assumption can be dropped. Our Theorem 2.3 says yes (for the indirect Galois correspondence). Note that $P P V$ extensions are a special case of Landesman strongly normal extensions.

3.5. Generalised strongly normal extensions. This is a direct transfer of the notions in Section 2 to the theory $\mathrm{DCF}_{0, m}$. In the paper [18] the second author introduced $X$-strongly normal extensions of a differential field $K$, in the ordinary context, where $X$ is an arbitrary $K$-definable set, also mentioning that things extend to the case of several derivations. The condition that $X\left(K^{\text {diff }}\right)=X(K)$ was included in the original definition, partly for consistency with the usual account of the Picard-Vessiot (or strongly normal) theory where the field of constants of $K$ is assumed to be algebraically closed, and partly so that $A u t_{\delta}(L / K)$ is a definable group in the differentially closed field $K^{\text {diff }}$. But in fact the condition that $X\left(K^{\text {diff }}\right)=X(K)$, can be dropped from Definition 2.1 of [18], still preserving the Galois correspondence (but now indirect). We briefly explain: 
Working now in $\mathrm{DCF}_{0, m}$, given a differential field $K$, and $K$-definable set $X$, we make:

DEFINITION 3.3. The differential field $L$ is an $X$-strongly normal extension of $K$, if $L$ is finitely generated over $K$, for each automorphism $\sigma$ of $\mathcal{U}$ over $K, \sigma(L) \subseteq L\langle X\rangle$, and $d c l(K, X) \cap L=K$.

This is precisely Definition 2.1 of [18], adapted to several derivations, and minus part (i). On the other hand, this also states that $q$ and $X$ satisfy conditions (I) and (II) from Section 2, where $q$ is the type over $K$ of some generator of $L$ over $K$. With this definition, Theorem 2.3 gives the Galois group, as well as the (indirect) Galois correspondence. The Galois group $G$ is a differential algebraic group, defined over $K$, and the Galois correspondence is between $K$-definable subgroups of $G$ and differential fields between $K$ and $L$.

3.6. Logarithmic differential equations on algebraic $\delta$-groups. This is the only section of the paper which may require some additional background from the reader. In the $m=1$ case, when $K$ is algebraically closed, the generalized strongly normal extensions of [18] come from "logarithmic differential equations on algebraic $\delta$-groups", as explained in [19]. This was suitably generalized by the first author to the $m>1$ case in [12]. It is worth mentioning that the differential Galois extensions coming from logarithmic differential equations on algebraic $\delta$-groups (over $K$ ) are all generalized strongly normal extensions of $K$ in the sense of Section 3.5. Thus, the Galois correspondence in this case is subsumed again by Theorem 2.3.

In [1] Daniel Bertrand and the second author study a special class of such algebraic $\delta$-groups and logarithmic differential equations on them, socalled "almost semiabelian $\delta$-groups", as part of a long term research project around functional Lindemann-Weierstrass theorems for families of semiabelian varieties. Conjecture 2.3 from [1] says roughly that the Galois group can be identified from the original equation via "gauge transformations over $K$ ". The aim here is to give a simple restatement of this property in terms of $X$-strongly normal extensions of $K$ (from Definition 3.2), for suitable $X$.

Here we assume $m=1$. We refer the reader to [19] or [1] but we repeat some definitions. By an algebraic $\delta$-group over $K$ we mean an algebraic group $G$ over $K$ together with a lifting of the derivation $\delta$ on $K$ to a derivation $s$ on the structure sheaf of $G$. We can and will identify $s$ with a homomorphic rational section over $K$ of the shifted tangent bundle $T_{\delta}(G) \rightarrow G$. The logarithmic derivative $d \log _{(G, S)}: G \rightarrow L G$ (to the Lie algebra of $G$ ) is the differential algebraic map taking $x \in G$ to $\delta(x) \cdot s(x)^{-1}$ computed in the algebraic group $T_{\delta}(G)$. A logarithmic differential equation on $(G, s)$ over $K$ is an equation of the form $d \log _{(G, s)}(-)=A$, where $A \in L G(K)$. When $G=G L_{n}$ and $s$ is the 0 -section of $T(G) \rightarrow G$, such an equation is simply a linear differential equation in matrix form.

$(G, s)^{\partial}$ denotes $\{x \in G: s(x)=\delta(x)\}$, the "kernel" of the $d \log _{(G, s)}$-map. It is a finite-dimensional differential algebraic group. $K_{(G, s)}^{\sharp}$ denotes the differential field generated over $K$ by $G^{\partial}\left(K^{\text {diff }}\right)$, the points of $G^{\partial}$ in the 
differential closure $K^{\text {diff }}$ of $K$. As any two solutions $y_{1}, y_{2} \in G\left(K^{\text {diff }}\right)$ of a logarithmic differential equation $d \log _{(G, s)}(-)=A$ on $(G, s)$ over $K$, differ by an element of $G^{\partial}\left(K^{\text {diff }}\right)$, it follows that $\operatorname{tr} \cdot \operatorname{deg}\left(K_{(G, s)}^{\sharp}(y) / K_{(G, s)}^{\sharp}\right)$ is the same, for any solution of the equation in $G\left(K^{\text {diff }}\right)$.

We write the lemma below for the case where $G$ is commutative and use additive notation, although suitably written it holds in general.

Lemma 3.4. Let $(G, s)$ be a commutative connected algebraic $\delta$-group over $K$ where $K$ is algebraically closed. Let $A \in L G(K)$ and let $y \in G\left(K^{\text {diff }}\right)$ be a solution of the equation

$$
(*) \quad \operatorname{dlog}_{(G, s)}(-)=A .
$$

Then the following are equivalent:

(i) tr.deg $\left(K_{(G, s)}^{\sharp}(y) / K_{(G, s)}^{\sharp}\right)$ equals $\min \{\operatorname{dim}(H): H$ is a connected algebraic $\delta$-subgroup of $G$ defined over $K$ and $A \in L(H)+$ $\left.d \log _{(G, s)}(G(K))\right\}$.

(ii) For some solution $y_{0}$ of $(*), K\left(y_{0}\right)$ is a $G^{\partial}$-strongly normal extension of $K$.

Proof. The condition in (i) that $A \in L(H)+d \log _{(G, s)}(G(K))$ is obviously equivalent to $y \in H+G(K)+G^{\partial}\left(K^{\text {diff }}\right)$ and will be used that way.

(i) $\Rightarrow$ (ii): Fix a solution $y$ of $(*)$. Suppose that $H$ is a connected algebraic $\delta$-subgroup of $G$ such that $y \in H+G(K)+G^{\partial}\left(K^{\text {diff }}\right)$ and

$$
\operatorname{dim}(H)=\operatorname{tr} . \operatorname{deg} .\left(K_{(G, s)}^{\sharp}(y) / K_{(G, s)}^{\sharp}\right) .
$$

So there is $y_{0} \in H+G(K)$ of the form $y+g$ for some $g \in G^{\partial}\left(K^{\text {diff }}\right)$. Note that $y_{0}$ is also a solution of $\left(^{*}\right)$ and moreover $\operatorname{tr} \cdot \operatorname{deg}\left(K\left(y_{0}\right) / K\right) \leq \operatorname{dim}(H)$. On the other hand, tr.deg. $\left(K_{(G, s)}^{\sharp}\left(y_{0}\right) / K_{(G, S)}^{\sharp}\right)=\operatorname{tr}$.deg. $\left(K_{(G, S)}^{\sharp}(y) / K_{(G, s)}^{\sharp}\right)=$ $\operatorname{dim}(H)$, by assumption. The conclusion is that $\operatorname{tr} \cdot \operatorname{deg}\left(K\left(y_{0}\right) / K\right)=\operatorname{dim}(H)$ and $y_{0}$ is independent from $K_{G}^{\sharp}$ over $K$ (in $\left.\mathrm{DCF}_{0}\right)$. As $K_{G}^{\sharp}=K\left(G^{\partial}\left(K^{\text {diff }}\right)\right.$ ), the latter says that $y_{0}$ is independent from $G^{\partial}\left(K^{\text {diff }}\right)$ over $K$, and this says that $y_{0}$ is independent from $G^{\partial}$ over $K$. As $K$ is algebraically closed and $G^{\partial}$ is definable, this says that $t p\left(y_{0} / K\right)$ has a unique extension to a complete type over $K \cup G^{\partial}$ which is precisely condition (II). As $y_{0}$ is a solution of $\left(^{*}\right)$ we have condition (I) too.

Conversely, suppose (ii) holds, witnessed by $y$. By (II), $y$ is independent of $G^{\partial}$ over $K$. By [1], Remark following Fact 2.2, let $H$ be a connected algebraic $\delta$-subgroup of $G$, over $K$ such that $H^{\partial}=\operatorname{Aut}\left(Y / K, G^{\partial}\right)$. Consider the orbit $y+H^{\partial}$. It is defined over $K^{\sharp}$, and over $K(y)$ (in $\mathrm{DCF}_{0}$ ), so also over $K$. But then $y+H$, the Zariski closure of $y+H^{\partial}$ is defined over $K$ (in $\mathrm{DCF}_{0}$ ). By elimination of imaginaries in ACF, let $e$ be a code of $y+H$ (in $\mathrm{ACF})$. But then $e \in K$. So $y+H$ is defined over $K$ in ACF so has a $K$ rational point $y_{1} \in G(K)$. So $y \in H+G(K)$. The minimality of $\operatorname{dim}(H)$ is clear.

Conjecture 2.3 from [1] can now be restated as: Let $G$ be an almost semiabelian $\delta$-group over $K=\mathbb{C}(t)^{\text {alg }}$, let $A \in L G(K)$, and consider the 
equation $\operatorname{dlog}_{(G, S)}(-)=A$. Then the equation has a solution $y$ such that $K(y)$ is a $G^{\partial}$-strongly normal extension of $K$.

§4. Acknowledgment. Anand Pillay was supported by NSF grant DMS1360702 .

\section{REFERENCES}

[1] D. Bertrand and A. Pillay, Galois theory, functional Lindemann-Weierstrass, and Manin maps. Pacific Journal of Mathematics, vol. 281 (2016), no. 1, pp. 51-82.

[2] Q. BRouetTe and F. PoinT. On Galois groups of strongly normal extensions, preprint, 2015, arXiv:1512.95998v1.

[3] P. J. CASSIDY and M. F. SINGER. Galois theory of parameterized differential equations and linear differential algebraic groups, Differential Equations and Quantum Groups (D. Bertrand, B. Enriquez, C. Mitschi, C. Sabbah, and R. Schaefke, editors), IRMA Lectures in Mathematics and Theoretical Physics, vol. 9, EMS Publishing House, Zürich, Switzerland, 2006, pp. 113-157.

[4] T. Crespo, Z. Hajto, and E. Sowa-Adamus, Galois correspondence theorem for Picard-Vessiot extensions. Arnold Mathematical Journal, vol. 2 (2016), no. 1, pp. 21-27.

[5] T. DycKerhoff, The inverse problem of differential Galois theory over the field $R(t)$, preprint, 2008, arXiv:0802.2897v1.

[6] H. Gillet, S. Gorchinskiy, and A. Ovchinnikov, Parametrized Picard-Vessiot extensions and Atiyah extensions. Advances in Mathematics, vol. 238 (2013), pp. 322-411.

[7] E. Hrushovski, Unidimensional theories are superstable. Annals of Pure and Applied Logic, vol. 50 (1990), pp. 117-138.

[8] E. Hrushovski, Computing the Galois group of a linear differential equation, Differential Galois Theory (S. Janeczko, editor), Banach Center Publications, vol. 59, Polish Academy of Sciences, Warszawa, Poland, 2012, pp. 97-138.

[9] M. Kamensky, Definable groups of automorphisms (Summary of Ph.D. thesis). https://www.math.bgu.ac.il/ kamenskm/.

[10] E. Kolchin, Differential Algebra and Algebraic Groups, Academic Press, New York, 1973.

[11] P. Landesman, Generalized differential Galois theory. Transactions of the American Mathematical Society, vol. 360 (2008), pp. 4441-4495.

[12] O. León SÁnchez, Relative D-groups and differential Galois theory in several derivations. Transactions of the American Mathematical Society, vol. 367 (2015), no. 11, pp. 7613-7638.

[13] O. León SÁnchez and J. Nagloo, On parameterized differential Galois extensions. Journal of Pure and Applied Algebra, vol. 220 (2016), no. 7, pp. 2549-2563.

[14] A. R. Magid, Differential Galois theory. Notices of the AMS, vol. 46 (1999), no. 9, pp. 1041-1049.

[15] D. Marker, M. Messmer, and A. Pillay, Model Theory of Fields, Lectures Note in Logic, vol. 5, Association of Symbolic Logic, Diego, CA, USA, 1996.

[16] A. Medvedev and R. TaKLoo-Bigash, An invitation to model-theoretic Galois theory. Bulletin of Symbolic Logic, vol. 16 (2010), no. 2, pp. 261-269.

[17] T. MCGraIL, The model theory of differential fields with finitely many commuting derivations. Journal of Symbolic Logic, vol. 65 (2000), no. 2, pp. 885-913.

[18] A. Pillay, Differential Galois theory I. Illinois Journal of Mathematics, vol. 42 (1998), no. 4, pp. 678-699.

[19] - Algebraic D-groups and differential Galois theory. Pacific Journal of Mathematics, vol. 216 (2004), pp. 343-360.

[20] - Geometric Stability Theory, Oxford University Press, Oxford, UK, 1996.

[21] - Around differential Galois theory, Algebraic Theory of Differential Equations (M. A. H. MacCallum and A. V. Mikhailov, editors), LMS Lecture Notes series, Cambridge University Press, Cambridge, UK, 2009, pp. 232-239. 
[22] M. van DER PUT and M. F. Singer, Galois Theory of Linear Differential Equations, Grundlehren der mathematischen Wissenschaften, vol. 328, Springer, New York, USA, 2003.

[23] B. Poizat, Une théorie de Galois imaginaire. Journal of Symbolic Logic, vol. 48 (1983), no. 4, pp. 1151-1170.

[24] B. PoIZAT, A Course in Model Theory: An Introduction to Contemporary Mathematical Logic, Springer, New York, USA, 2000.

[25] B. I. ZILBER, Totally categorical theories: Structural properties and non-finite axiomatizability, Model Theory of Algebra and Arithmetic (L. Pacholski, J. Wierzejewski, and A. Wilkie, editors), Lecture Notes in Mathematics, vol. 834, Springer-Verlag, Berlin, Heidelberg, New York, 1980, pp. 381-410.

\author{
SCHOOL OF MATHEMATICS \\ UNIVERSITY OF MANCHESTER \\ OXFORD ROAD \\ MANCHESTER M13 9PL, UK \\ E-mail: omar.sanchez@manchester.ac.uk \\ DEPARTMENT OF MATHEMATICS \\ UNIVERSITY OF NOTRE DAME \\ 255 HURLEY, NOTRE DAME \\ IN 46556, USA \\ E-mail: apillay@nd.edu
}

